\title{
Perfil nutricional por antropometría de niños/as menores de 5 años del sistema público de salud, 2013
}

\section{Anthropometry Nutritional Profile of Children under 5 years of Public Health System, 2013}

\author{
Geraldine Morinigo Isla ${ }^{(1)}$, Susana Sánchez Bernal ${ }^{(1)}$, Vania Sispanov Pankow ${ }^{(1)}$, Gonzalo Rolón \\ Villalba $^{(1)}$, Carolina Bonzi Arévalos ${ }^{(1)}$, Hugo Medina ${ }^{(1)}, K$ atherine Cardozo ${ }^{(1)}$, Alicia Rodas Congo ${ }^{(1)}$, \\ Mariela Brizuela Rivarola ${ }^{(1)}$, Laura Mendoza de Arbo ${ }^{(1)}$
}

\section{RESUMEN}

Introducción: Es fundamental realizar vigilancia de la situación nutricional de niños y niñas menores de cinco años para realizar intervenciones oportunas ante casos de malnutrición. Objetivo: Determinar la situación nutricional de niños y niñas menores de cinco años, usuarios de servicios públicos de salud. Materiales y Métodos: Estudio transversal, descriptivo, analítico, realizado con datos del Sistema de Vigilancia Nutricional en servicios del Ministerio de Salud. El diagnóstico nutricional fue por antropometría, según criterios de la OMS. Resultados: Fueron evaluados 52.784 niños y niñas menores de cinco años, edad promedio 21 meses (1-59 meses), 49,5\% varones. Los promedios zPeso/Edad, zPeso/Talla y zTalla/Edad fueron -0,12 $\pm 1,17 \mathrm{DE}$; $0,19 \pm 1,42 \mathrm{DE} ;-0,41 \pm 1,42$ DE respectivamente. Se observó 4,8\% de Desnutrición Global (DG) (zPeso/Edad<-2DE), 5,4\% Desnutrición Aguda (DA) (zPeso/Talla<-2DE) y 12,2\% Desnutrición Crónica (DC) (zTalla/Edad<-2DE). Hubo mayor desnutrición en niños/as: residentes en área rural (DG 5,1 rural vs 4,5\% urbano; DA5,7 rural vs 5,2\% urbano; DC 13,3 rural vs $11,1 \%$ urbano; $\chi 2 \mathrm{p}<0,0001)$, con antecedentes de bajo peso de nacimiento(DG $13,9<2500 \mathrm{~g}$ vs $4,1 \% \geq 2500$ g; DA $9,7<2.500$ g vs $5,1 \% \geq 2500$ g; DC $22,9<$ $2.500 \mathrm{~g}$ vs $11,3 \% \geq 2500 \mathrm{~g} ; \chi 2 \mathrm{p}<0,0001)$, hijos de madres con $<6$ años de escolaridad (DG 6,9 $<6$ años vs $4,3 \% \geq 6$ años; DA 6,2 $<6$ años vs $5,3 \% \geq 6$ años; DC $16,3<6$ años vs $11,2 \% \geq 6$ años; $\chi 2$ $\mathrm{p}<0,0001$ ), niños (DG 5,1 niños vs 4,5\% niñas; DC 13,5 niños vs $10,9 \%$ niñas; $\chi 2 \mathrm{p}<0,0001)$. Presentaron obesidad (zPeso/Talla >2DE) el 9,2\%. Conclusiones: Hubo malnutrición tanto por déficit, a expensas de la desnutrición crónica, como por exceso. La desnutrición estuvo asociada a la procedencia rural, bajo peso de nacimiento, baja escolaridad materna y al sexo masculino.

Palabras clave: Estado nutricional, trastornos de la nutrición del niño, desnutrición.

\section{ABSTRACT}

Introduction: It is essential to conduct surveillance of the nutritional status of children under 5 years to make early interventions in cases of malnutrition. Objective: To determine the nutritional status of children under 5 years, users of public health services. Materials and Methods: Cross-sectional, descriptive, analytical, made with System data Nutritional Surveillance services of the Ministry of Health Study. The nutritional diagnosis was by anthropometry, according to WHO criteria. Results: We evaluated 52.784 children under 5 years, average age 21 months (1-59 months), $49.5 \%$ male. The zWeight/Age, zWeight/ Height and zHeight/Age averages were $-0.12 \pm$ $1,17 \mathrm{DE} ; 1,42 \mathrm{DE} \pm 0.19 ;-0.41 \pm 1.42$ respectively. $4.8 \%$ of Global underweight (DG) (zWeight/Age $<-2$ SD), 5,4\% acute underweight (DA) (zWeight/Height $<-2$ SD) and $12.2 \%$ short height (DC) (zHeight/Age <-2 SD). There was increased malnutrition in children: rural residents (DG 5.1 rural area vs. $4.5 \%$ urban, DA 5.7 rural area vs. 5.2\% urban, DC 13.3 rural area vs. $11.1 \%$ urban; $\chi 2 \mathrm{p}<0.0001$ ), with a history of low birth weight (DG $13.9<2500 \mathrm{~g}$ vs. $4.1 \%$ $\geq 2500$ g; DA $9.7<2500$ g vs. $5.1 \% \geq 2500$ g, DC $22.9<2500 \mathrm{~g}$ vs. $\geq 2500 \mathrm{~g} 11.3 \%$; $\chi 2 \mathrm{p}<0.0001)$, children of mothers with $<6$ years of schooling ( DG $6.9<6$ years vs. $4.3 \% \geq 6$ years ; DA $6.2<6$ years vs. $5.3 \% \geq 6$ years; DC $16.3<6$ years vs. $11.2 \% \geq 6$ years; $\chi 2 \mathrm{p}<0.0001$ ), male sex (DG 5.1 male vs. $4.5 \%$ female; DC 13,5 male vs. $10.9 \%$ female; $\chi 2 p<0.0001$ ). They presented obesity (zWeight/Height > 2SD) $9.2 \%$. Conclusions: There was malnutrition for deficit at the expense of short height and excess. Underweight was associated with rural area, low birth weight, low maternal education and male sex.

Keywords: Nutritional Status, Child Nutrition Disorders, Malnutrition.

\footnotetext{
1. Instituto Nacional de Alimentación y Nutrición, Ministerio de Salud Pública y Bienestar Social. Asunción, Paraguay. Correspondencia: Geraldine Morinigo Isla. E-mail: geralmorinigo@gmail.com, Susana Sánchez Bernal. E-mail: susana.sanchez.ber@gmail.com 


\section{INTRODUCCIÓN}

Los primeros años de vida de los niños son de vital importancia, ya que tanto el cerebro, como el sistema nervioso alcanzan un $25 \%$ del tamaño final al momento del nacimiento y hasta un $70 \%$ del crecimiento máximo durante el primer año de vida, razón por la cual una adecuada nutrición durante la primera infancia posee mayores beneficios y la carencia en esta etapa puede causar daños físicos y cerebrales irreversibles ${ }^{(1,2)}$. La Convención sobre los Derechos del Niño establece el derecho de todos los niños a un nivel de vida adecuado para su desarrollo físico, mental, espiritual, moral y social ${ }^{(3)}$.

El estado nutricional de los menores de cinco años ha sido reconocido como un valioso indicador que refleja el estado de desarrollo de una población. En la actualidad en América Latina y el Caribe, grandes grupos poblacionales aún viven en situación de inseguridad alimentaria y sufren de desnutrición crónica, según estimaciones de la Organización Mundial de la Salud, en los países en desarrollo con alta mortalidad, la desnutrición infantil es causa del $15 \%$ de los años de vida perdidos por muerte o $\operatorname{discapacidad}^{(4,5)}$.

La desnutrición en niños y niñas menores de cinco años es uno de los efectos más directos de la carencia de alimentos, y tiene como consecuencia la transmisión generacional de la pobreza extrema y la desigualdad ya que produce impactos negativos en la salud, la educación y la productividad de la población ${ }^{(6)}$.

Por ello, es de relevancia la detección precoz a fin de minimizar las consecuencias, principalmente en el crecimiento y desarrollo del niño. En Paraguay a partir del año 2000 se implementa el Sistema de Vigilancia Alimentario Nutricional (SISVAN), que busca conocer la situación nutricional de poblaciones vulnerables, entre ellas menores de cinco años, a fin de encaminar intervenciones nutricionales y lograr romper el círculo de la pobreza.

El objetivo de este trabajo fue determinar el estado nutricional de niños y niñas menores de cinco años que asisten a servicios de salud pública e identificar los factores asociados a desnutrición.

\section{MATERIALES Y MÉTODOS}

Diseño: estudio de corte transversal, descriptivo con componente analítico. El proceso de muestreo fue no probabilístico de casos consecutivos. La población estudiada fueron niños y niñas menores de cinco años que asistieron a servicios de salud pública a nivel país, en el periodo de tiempo comprendido entre el enero a diciembre 2013.

Fueron incluidos los niños y niñas con edad comprendida entre 1 y $<60$ meses que asistieron a los servicios de salud para su control, que fijaron residencia en el área de influencia de la Región Sanitaria y cuyas madres estuvieron dispuestas a colaborar para la realización de la encuesta.

Las variables estudiadas fueron: estado nutricional, sexo, procedencia, peso de nacimiento y escolaridad materna.

Se analizaron datos de menores de cinco años generados por el Sistema de Vigilancia Alimentaria y Nutricional-SISVAN en servicios de salud de las 18 regiones sanitarias. Fueron aplicados los criterios de evaluación nutricional de la OMS para puntaje $\mathrm{z}$ Peso/Edad (zP/E), z Peso/Talla (zP/T), z Talla/Edad (zT/E).

Referente al análisis estadístico, los datos fueron consignados en una planilla de Excel versión 5.0 pre codificada y analizados con el programa WHO ANTRO y EPIINFO 6.04. Las variables continuas se expresaron como medias y desviación estándar según su ajuste a la normalidad. Las variables categóricas se expresaron como porcentajes.

Para determinar la asociaciones se utilizó la prueba de chi cuadrado, considerando significativo un error alfa menor a 0,05 $(p<0,05)$. La fuerza de asociación entre las variables se midió por el Odds Ratio(OR).

\section{RESULTADOS}

La población total analizada fue de 52.784 niños, el $50,4 \%$ correspondió al sexo femenino $(n=26.648)$ y el $49,6 \%$ al sexo masculino ( $n=26.137)$. El $62 \%$ de los niños fueron menores de dos años de edad. La edad promedio fue de $21,3 \pm 16,6$ meses.

La prevalencia de desnutrición global (zP/E<-2 DE) 
en las diferentes regiones sanitarias en el año 2013 en la población de niños fue del $4,8 \%(\mathrm{n}=2.529)$ y la desnutrición aguda $(\mathrm{zP} / \mathrm{T}<-2 \mathrm{DE})$ fue de $5,4 \%$ $(\mathrm{n}=2.869)$. Asimismo, la desnutrición crónica $(\mathrm{zT} / \mathrm{E}<-$ 2 DE) alcanzó la cifra de 12,2\% (n=6.437) y la obesidad $(\mathrm{zP} / \mathrm{T}>2 \mathrm{DE})$, tabla 1.

Tabla 1. Situación nutricional de niños y niñas menores de 5 años que asistieron a servicios de salud pública-Paraguay 2013.

\begin{tabular}{lcccc}
\hline Regiones Sanitarias & $\begin{array}{c}\text { Desnutrición } \\
\text { Global } \%\end{array}$ & $\begin{array}{c}\text { Desnutrición } \\
\text { Aguda } \%\end{array}$ & $\begin{array}{c}\text { Desnutrición } \\
\text { Crónica } \%\end{array}$ & $\begin{array}{c}\text { Obesidad } \\
\text { \% }\end{array}$ \\
\hline 1- Concepción & 8,1 & 5,8 & 22,6 & 7,7 \\
\hline 2- San Pedro & 6,7 & 7,5 & 13,1 & 5,3 \\
\hline 3- Cordillera & 2,6 & 4,8 & 11,9 & 13,6 \\
\hline 4- Guairá & 6,0 & 7,2 & 14,7 & 7,3 \\
\hline 5- Caaguazú & 5,2 & 6,6 & 13,7 & 6,2 \\
\hline 6- Caazapá & 5,8 & 4,2 & 16,2 & 6,7 \\
\hline 7- Itapúa & 4,0 & 3,8 & 11,5 & 10,1 \\
\hline 8- Misiones & 1,9 & 1,7 & 9,4 & 15,2 \\
\hline 9- Paraguarí & 2,4 & 2,8 & 10,1 & 13,5 \\
\hline 10- Alto Paraná & 5,4 & 6,0 & 14,6 & 10,6 \\
\hline 11- Central & 5,9 & 7,0 & 11,2 & 8,3 \\
\hline 12- Ñeembucú & 1,6 & 2,4 & 6,1 & 11,1 \\
\hline 13- Amambay & 5,0 & 3,3 & 10,6 & 6,2 \\
\hline 14- Canindeyú & 5,4 & 5,9 & 13,7 & 6,9 \\
\hline 15- Pdte. Hayes & 3,4 & 3,5 & 10,1 & 10,4 \\
\hline 16- Boquerón & 2,8 & 3,2 & 11,1 & 10,6 \\
\hline 17- Alto Paraguay & 2,4 & 1,2 & 2,4 & 1,2 \\
\hline 18- Capital & 2,9 & 5,0 & 11,7 & 15,8 \\
\hline Total general & $\mathbf{4 , 8}$ & $\mathbf{5 , 4}$ & $\mathbf{1 2 , 2}$ & $\mathbf{9 , 2}$ \\
\hline SISvAN- INAN-MSPYBS & & & & \\
\hline
\end{tabular}

Al comparar la situación nutricional entre géneros se pudo observar que los niños de sexo masculino presentaron significativamente mayor prevalencia de desnutrición global y crónica (Tabla 2). A su vez los niños y niñas de zonas rurales se encontraron significativamente más desnutridos que los de zonas urbanas (Tabla 3).

Tabla 2. Desnutrición infantil por Género.

\begin{tabular}{lccccccc}
\hline Genero & \multicolumn{2}{c}{ Global } & \multicolumn{2}{c}{ Aguda } & \multicolumn{2}{c}{ Crónica } & Total \\
& $\mathbf{n}$ & $\mathbf{\%}$ & $\mathbf{n}$ & $\mathbf{\%}$ & $\mathbf{n}$ & $\mathbf{\%}$ & General \\
\hline Femenino & 1.189 & 4,5 & 1.421 & 5,3 & 2.899 & 10,9 & 26.647 \\
Masculino & 1.340 & $5,1^{*}$ & 1.448 & 5,5 & 3.538 & $13,5^{*}$ & 26.137 \\
Total general & $\mathbf{2 . 5 2 9}$ & $\mathbf{4 , 8}$ & $\mathbf{2 . 8 6 9}$ & $\mathbf{5 , 4}$ & $\mathbf{6 . 4 3 7}$ & $\mathbf{1 2 , 2}$ & $\mathbf{5 2 . 7 8 4}$ \\
\hline *Prueba de Chi cuadrado; & $\mathrm{p}=0,000001$ & & & & &
\end{tabular}

Tabla 3. Desnutrición en niños y niñas menores de cinco años, por Zona.

\begin{tabular}{lccccccc}
\hline Zona & \multicolumn{2}{c}{ Global } & \multicolumn{2}{c}{ Aguda } & \multicolumn{2}{c}{ Crónica } & Total \\
& $\mathbf{n}$ & $\mathbf{\%}$ & $\mathbf{n}$ & $\mathbf{\%}$ & $\mathbf{n}$ & $\mathbf{\%}$ & General \\
\hline Rural & 1.349 & $5,1 *$ & 1.515 & $5,7^{*}$ & 3525 & $13,3^{*}$ & 26.525 \\
Urbana & 1.180 & 4,5 & 1.354 & 5,2 & 2912 & 11,1 & 26.259 \\
Total general & $\mathbf{2 . 5 2 9}$ & $\mathbf{4 , 8}$ & $\mathbf{2 . 8 6 9}$ & $\mathbf{5 , 4}$ & $\mathbf{6 . 4 3 7}$ & $\mathbf{1 2 , 2}$ & $\mathbf{5 2 . 7 8 4}$ \\
\hline *Prueba de Chi cuadrado; & $\mathrm{p}=0,000001$ & & & & &
\end{tabular}

Se encontró asociación significativa entre el bajo peso de nacimiento y la desnutrición global, aguda y crónica (Tabla 4). Al contrastar el estado nutricional con los años de estudio materno, se encontraron significativamente más desnutridos los niños y niñas de madres con menos de 6 años de estudio (Tabla 5).

Tabla 4. Desnutrición en niños y niñas menores de cinco años, por Peso de Nacimiento.

\begin{tabular}{lccccccc}
\hline Peso de & \multicolumn{2}{c}{ Global } & \multicolumn{2}{c}{ Aguda } & \multicolumn{2}{c}{ Crónica } & Total \\
Nacimiento & $\mathbf{n}$ & $\mathbf{\%}$ & $\mathbf{n}$ & $\mathbf{\%}$ & $\mathbf{n}$ & $\mathbf{\%}$ & General \\
\hline Bajo & 516 & 13,9 & 359 & 9,7 & 846 & 22,9 & 3.701 \\
Adecuado & 2.013 & 4,1 & 2.510 & 5,1 & 5.591 & 11,3 & 49.083 \\
\hline Total general & $\mathbf{2 . 5 2 9}$ & $\mathbf{4 , 8}$ & $\mathbf{2 . 8 6 9}$ & $\mathbf{5 , 4}$ & $\mathbf{6 . 4 3 7}$ & $\mathbf{1 2 , 2}$ & $\mathbf{5 2 . 7 8 4}$ \\
\hline *Prueba de Chi cuadrado; $\mathrm{p}=0,000001$ & & & & &
\end{tabular}

Tabla 5. Desnutrición en niños y niñas menores de cinco años por Escolaridad Materna.

\begin{tabular}{lccccccc}
\hline Escolaridad & \multicolumn{2}{c}{ Global } & \multicolumn{2}{c}{ Aguda } & \multicolumn{2}{c}{ Crónica } & Total \\
materna & $\mathbf{n}$ & $\mathbf{\%}$ & $\mathbf{n}$ & $\mathbf{\%}$ & $\mathbf{n}$ & $\mathbf{\%}$ & General \\
\hline$<6$ años de estudio & 695 & 6,9 & 624 & 6,2 & 1.634 & 16,3 & 10.035 \\
$>6$ años de estudio & 1.834 & 4,3 & 2.245 & 5,3 & 4.803 & 11,2 & 42.749 \\
\hline Total general & $\mathbf{2 . 5 2 9}$ & $\mathbf{4 , 8}$ & $\mathbf{2 . 8 6 9}$ & $\mathbf{5 , 4}$ & $\mathbf{6 . 4 3 7}$ & $\mathbf{1 2 , 2}$ & $\mathbf{5 2 . 7 8 4}$ \\
\hline *Prueba de Chi cuadrado; $\mathrm{p}=0,000001$ & & & & &
\end{tabular}

\section{DISCUSIÓN}

La desnutrición, al afectar a la capacidad intelectual y cognitiva del niño, disminuye su rendimiento escolar y el aprendizaje, limitando la capacidad del niño de convertirse en un adulto que pueda contribuir, al progreso de su comunidad y de su país ${ }^{(3)}$.

Según resultados de la Encuesta Permanente de Hogares de Paraguay del año $2005^{(7)}$, la prevalencia de desnutrición global en niños y niñas menores de 5 años de edad fue del 3,4\% con los nuevos estándares de crecimiento de la OMS, dato similar a lo registrado en este trabajo, donde la desnutrición global alcanza el 4,8\%, no obstante el riesgo de desnutrición representa el 18,1\%.

Por otro lado la desnutrición crónica, a veces invisible y con menor atención, sigue siendo un problema de mayor magnitud en cuanto al número de niños afectados ${ }^{(3)}$, las cifras de desnutrición crónica casi triplican las de desnutrición global, siendo $12,2 \%$ en el presente estudio y $17,5 \%$ en la Encuesta Permanente de Hogares ${ }^{(7)}$. Sin embargo, los niños que se encontraron en situación de riesgo de talla baja presentaron una prevalencia del $21,7 \%$. 
Al tratarse de poblaciones indígenas, las cifras de desnutrición global se duplican al 9,8\% según las Encuesta a Hogares Indígenas $(\mathrm{EHI})^{(8)}$, y son aún más elevadas al tratarse de desnutrición crónica $(41,7 \%$ vs $12,2 \%)$, estos datos podrían estar indicando la situación de inequidad vinculada en mayor medida con un medio ambiente adverso y factores agresores $^{(9,10)}$.

La evolución de los últimos 5 años, según datos del SISVAN, refleja que la prevalencia de desnutrición global de niños que asisten a servicios de salud pública disminuyó del $6,2 \%$ al $4,8 \%$, así como las cifras de talla baja del 18,1\% al 12,2\%, lo cual podría atribuirse a la implementación del Programa Alimentario Nutricional Integral y también a la implementación de los servicios de atención $\operatorname{primaria}^{(11)}$.

Al disgregar la situación nutricional por regiones sanitarias/departamentos y sus distritos se presenta la situación de que en una misma región existen diferentes cifras de desnutrición, dependiendo de la zona, encontrándose asociación estadísticamente significativa entre niños y niñas que habitan en zonas rurales y la desnutrición, esto refleja la situación del país, donde el $32 \%$ de los niños y las niñas menores de cinco años de edad que viven en el área rural se encuentran en situación de extrema pobreza ${ }^{(12)}$.

Por otro lado en Latinoamérica, el sobrepeso y la obesidad en niños menores de 5 años, son de alguna manera subestimados, por ser considerados problemas propios de los países desarrollados y menos relevantes, sin embargo, el panorama regional de América Latina y el Caribe estima una prevalencia de entre $7 \%$ y $12 \%$ de obesidad, alcanzando un $9,1 \%$ en el presente estudio ${ }^{(13,14)}$.

Durante el ciclo vital, el bajo peso de nacimiento tiene repercusiones sobre el desarrollo físico e intelectual, asimismo aumenta el riesgo de mortalidad infantil y de desnutrición. Existe evidencia de que los niños con bajo peso al nacer tienen un riesgo casi cinco veces mayor de ser desnutridos que los nacidos con un peso adecuado, en el presente estudio se encontró una prevalencia de bajo peso del 6,6\%, habiendo asociación significativa entre el bajo peso al nacer y la desnutrición ${ }^{(6,15)}$.

También la educación materna adquiere un papel importante con el estado de salud de una población y/o un individuo, por lo resulta un factor crítico para el desarrollo infantil, a mayor escolaridad será posible comprender la importancia del mantenimiento de condiciones óptimas para la promoción de la salud, propios y de su descendencia, con estilos de vida saludables. En niños en situación de pobreza el mejor nivel educativo de la madre, constituyendo seis años de estudio en promedio, es un factor protector del estado nutricional en los niños menores de cinco años de edad, concordando con este estudio donde se encontró asociación significativa entre el la nula o baja escolaridad materna y la desnutrición ${ }^{(13,16,17)}$

En el presente estudio se han evidenciado como factores de riesgo de desnutrición en menores de 5 años, fijar residencia en zonas rurales, principalmente por condiciones de acceso y disponibilidad, el bajo peso de nacimiento, lo cual podría relacionarse con un estado nutricional materno deficiente, y uno de los determinantes sociales más importantes, la educación, sin educación no existe avance de la población. Por tanto, para lograr un crecimiento económico como País, y reducir la pobreza debemos realizar inversión en el capital humano, teniendo en cuenta estos y otros factores que condicionan el desarrollo de un capital humano eficiente.

\section{CONCLUSIÓN}

Hubo malnutrición en niños menores de cinco años de edad, tanto por déficit, a expensas de la desnutrición crónica, como por exceso. La desnutrición estuvo asociada a la procedencia rural, bajo peso de nacimiento, baja escolaridad materna y al sexo masculino. 


\section{REFERENCIAS}

1. Consejo Nacional de la Niñez y Adolescencia. Construir otro para Paraguay para niños, niñas y adolescentes. Asunción: Consejo Nacional de la Niñez y Adolescencia; 2005.

2. Villegas S, Ivanovic R, Pérez H, Almagià A, Urrutia M, Rodríguez M, Larraín C, Ivanovic, D. Peso de nacimiento y posterior estado nutricional, desarrollo cognitivo y actividad ocupacional: una revisión crítica. ALAN. 2009;59(4):369-77.

3. UNICEF. La desnutrición infantil: causas, consecuencias y estrategias para su prevención y tratamiento. Madrid: Fondo de las Naciones Unidas para la Infancia; 2011.

4. Buitrón D, Hurtig A, San Sebastián M. Estado nutricional en niños naporunas menores de cinco años en la Amazonía ecuatoriana. Rev Panam Salud Pública. 2004;15(3):151-59.

5. CEPAL. Inseguridad alimentaria y nutricional en América Latina y el Caribe: hambre y desnutrición en América Latina y el Caribe. Santiago de Chile: CEPAL; 2009.

6. CEPAL. Hambre y desnutrición en los países miembros de la Asociación de Estados del Caribe: el costo del hambre y la desnutrición. Santiago de Chile: CEPAL; 2005.

7. Sanabria M. Análisis de la situación de salud infantil y antropometría en menores de 5 años. Asunción: DGEECEPH;2005.

8. Sanabria M, Sánchez S, Medina N. Perfil nutricional de niños y niñas indígenas menores de 5 años de edad en el Paraguay: encuesta a hogares indígenas 2008. XLVI Reunión de la Sociedad Latinoamericana de Investigación Pediátrica SLAIP. Cusco, Perú. Noviembre; 2009.

9. CEPAL. Mortalidad infantil y en la niñez de pueblos indígenas y afro descendientes de América Latina: inequidades estructurales, patrones diversos y evidencia de derechos no cumplidos. Santiago: CEPAL; 2010.

10. Echaugüe G, Sosa L, Díaz V, Funes P, Pistilli N, Zenteno $\mathrm{J}$. Evaluación del estado nutricional en niños menores de 5 años de edad de comunidades rurales del Departamento de Caazapá. Asunción: Instituto de Investigaciones en Ciencias de la Salud; 2012.

11. Departamento de Monitoreo y Evaluación, Sistema de Vigilancia Alimentaria Nutricional (SISVAN). Dirección de Nutrición y Programas Alimentarios del Instituto Nacional de Alimentación y Nutrición (INAN). Situación nutricional de niños y niñas menores de 5 años que acudieron a los servicios de salud 2001-2012. Asunción: Ministerio de Salud Pública y Bienestar Social; 2012.

12. Lezcano L, Sanabria M. Perfil nutricional de niños y niñas beneficiarios de un programa alimentario, al ingreso y a los seis meses de tratamiento. Pediatr (Asunción). 2010;37(2):118-22.

13. Tazza R, Bullon L. ¿Obesidad o desnutrición? : problema actual de los niños peruanos menores de 5 años. An Fac Med. 2006; 67(3):214-23.

14. Organización Panamericana de la Salud. Salud en las Américas: panorama regional y perfiles de país. Washington CD;2012.

15. Alonso O, González D, Abreu G. Malnutrición proteico-energética en niños menores de 5 años. Rev Cubana Pediatr. 2007;79(2).

16. UNICEF. Estado Mundial de la Infancia 2001. Nueva York: UNICEF; 2001.

17. Sanabria M, Sánchez S. Factores protectores del estado nutricional de la niñez paraguaya. Revista de Análisis Economía \& Sociedad.2001;2(5):111-146. 